\title{
Radio linear and circular polarization from $\mathrm{M} \mathrm{81* \star}$
}

\author{
A. Brunthaler ${ }^{1,2}$, G. C. Bower ${ }^{3}$, and H. Falcke ${ }^{4,5}$ \\ 1 Joint Institute for VLBI in Europe, Postbus 2, 7990 AA Dwingeloo, The Netherlands \\ e-mail: brunthal@mpifr-bonn.mpg.de \\ 2 Max-Planck-Institut für Radioastronomie, Auf dem Hügel 69, 53121 Bonn, Germany \\ 3 Radio Astronomy Laboratory, University of California, Berkeley, CA 94720, USA \\ 4 ASTRON, Postbus 2, 7990 AA Dwingeloo, The Netherlands \\ 5 Department of Astrophysics, Radboud Universiteit Nijmegen, Postbus 9010, 6500 GL Nijmegen, The Netherlands
}

Received 30 December 2005 / Accepted 19 January 2006

\begin{abstract}
We present results from archival and new Very Large Array (VLA) data observations to investigate the long term behavior of the circular polarization of $\mathrm{M} 81^{*}$, the nuclear radio source in the nearby galaxy $\mathrm{M} 81$. We also used the Berkeley-Illinois-Maryland Association (BIMA) array to observe M 81* at 86 and $230 \mathrm{GHz}$. M 81* is unpolarized in the linear sense at a frequency as high as $86 \mathrm{GHz}$ and shows variable circular polarization at a frequency as high as $15 \mathrm{GHz}$. The spectrum of the fractional circular polarization is inverted in most of our observations. The sign of circular polarization is constant over frequency and time. The absence of linear polarization sets a lower limit to the accretion rate of $10^{-7} M_{\odot} \mathrm{y}^{-1}$. The polarization properties are strikingly similar to the properties of Sgr A*, the central radio source in the Milky Way. This supports the hypothesis that M 81* is a scaled up version of Sgr A*. On the other hand, the broad band total intensity spectrum declines towards milimeter wavelengths which differs from previous observations of M $81^{*}$ and also from Sgr A*.
\end{abstract}

Key words. galaxies: active - galaxies: individual: Messier Number: M 81 - polarization - radio continum: galaxies galaxies: nuclei

\section{Introduction}

The nearby spiral galaxy M 81 (NGC 3031) shares many properties with the Milky Way. It is similar in type, size and mass and it also contains a nuclear radio source, $\mathrm{M} 81^{*}$, that is most likely associated with a supermassive black hole. M $81^{*}$ has been studied extensively using Very Long Baseline Interferometry (VLBI) in the past. Bietenholz et al. (2000) resolved M 81* into a stationary core with a one sided jet. Multi-wavelength (Ho et al. 1996) and sub-millimeter observations (Reuter \& Lesch 1996) showed many similarities between M $81^{*}$ and $\mathrm{Sgr} \mathrm{A}^{*}$, the central radio source in our Milky Way (Melia \& Falcke 2001). A jet model of Sgr A* has been applied to $\mathrm{M} 81^{*}$, where it can reproduce the radio flux density and the size of the radio core by changing the accretion rate (Falcke 1996). The sizes of both radio sources show a $\sim 1 / v$ dependency on the frequency (e.g. Bietenholzet al. 2004 for M 81*; and Bower et al. 2004 and Shen et al. 2005, for Sgr A*).

M 81* is an apparent transitional object between Sgr A* and high luminosity AGN. As the brightest of the nearby low luminosity AGN (LLAGN), it is 5 orders of magnitude brighter than Sgr A* at radio wavelengths. M $81 *$ is substantially underluminous at X-ray wavelengths $\left(L \sim 10^{-5} L_{\text {edd }}\right)$, yet not as much as Sgr A* $\left(L \sim 10^{-10} L_{\text {edd }}\right)$. Still, it is the faintest LLAGN we can study.

Furthermore, the polarization properties of $\mathrm{M} 81^{*}$ and Sgr A* are very similar. Sgr A* shows circular polarization in absence of linear polarization (Bower et al. 1999a,b,c) and we

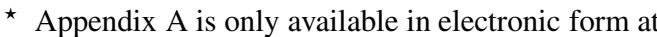
http://www . edpsciences . org
}

detected the same behaviour in M 81* (Brunthaler et al. 2001.) The polarization properties of $\mathrm{Sgr} \mathrm{A}^{*}$ and $\mathrm{M} 81^{*}$ are in contrast to the properties of most radio jets in active galactic nuclei where linear polarization often exceeds circular polarization by a large factor (e.g. Wardle et al. 1998; Rayner et al. 2000). The absence of linear polarized emission in Sgr A* can be explained as a consequence of the accretion flow.

Bower et al. (2002b) investigated the long term behavior of the circular polarization in Sgr A* from archival VLA data and showed that $i$ ) the circular polarization is variable on timescales of days to months, $i i)$ the sign of the circular polarization stayed constant over the entire time range of almost 20 years, and iii) the average spectrum of circular polarization is inverted.

After the discovery of circular polarization in M 81* we used the VLA to investigate the variability of the circular polarization on short timescales. We used additional archival VLA data to investigate the long term behavior of the circular polarization of $\mathrm{M} 81 *$.

\section{Observations}

\subsection{Archival VLA data}

M 81* and the supernova SN1993J in M 81 were observed many times with the Very Long Baseline Array (VLBA) and the phased Very Large Array (VLA) over the last decade (e.g. Bartel et al. 1994; Bietenholz et al. 2000). The observations were typically $16 \mathrm{~h}$ in duration, were made at different frequencies and involved rapid switching between M 81*, SN1993J and scans roughly every hour on the extragalactic background source $0954+658$ for calibration purposes. We used the VLA data from 

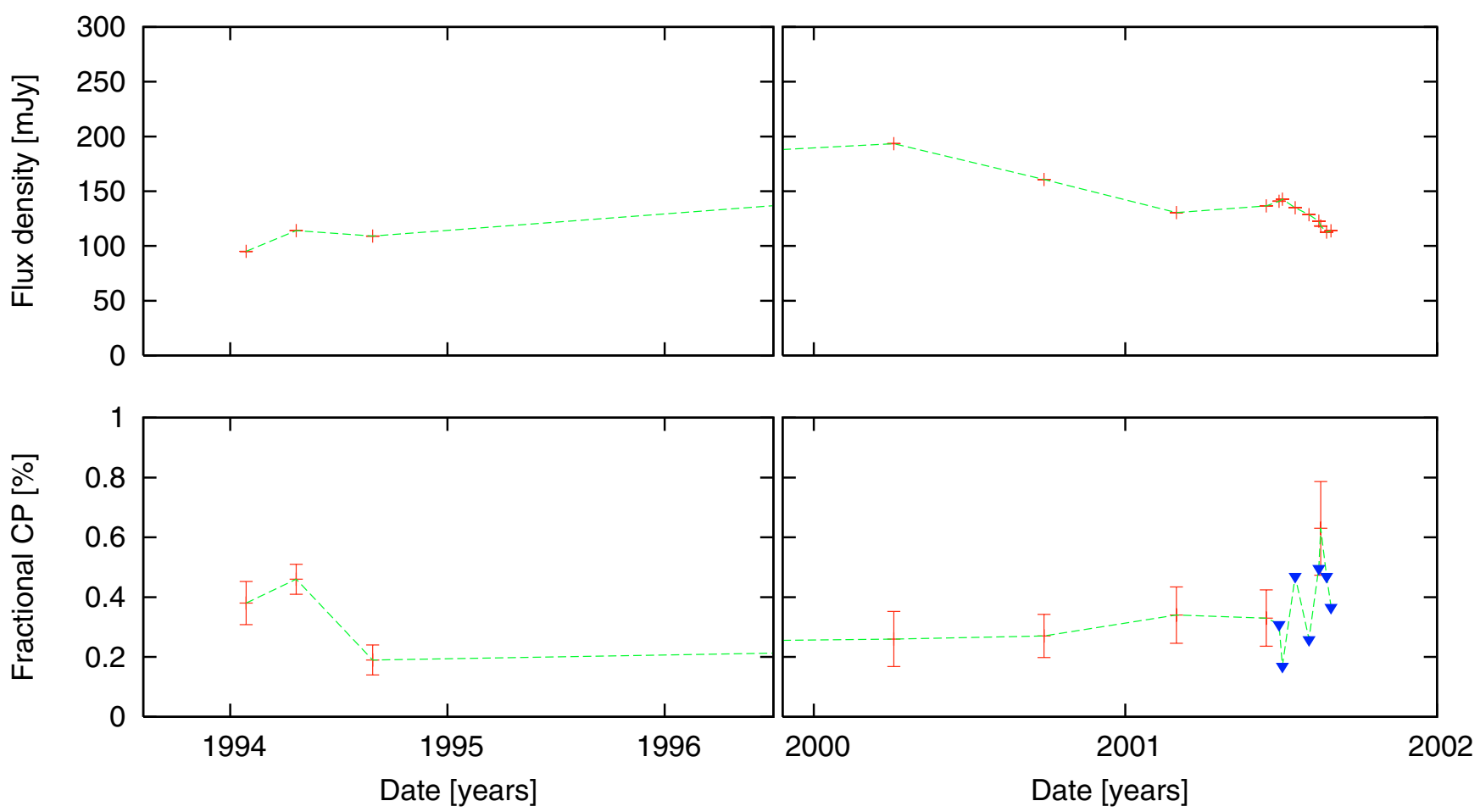

Fig. 1. Light-curve in total intensity (top) and fractional circular polarization of $\mathrm{M} 81^{*}$ at $4.8 \mathrm{GHz}$ for the archival data (left) and the new observations (right). The filled triangles are $3 \sigma$ upper limits. The two data points in 2000 are taken from Brunthaler et al. (2001).

the observations on 5 November $1993(v=8.4$ and $15 \mathrm{GHz})$, 16 December 1993 (8.4 and $15 \mathrm{GHz}), 29$ January 1994 (4.8 and $8.4 \mathrm{GHz}), 21$ April 1994 (4.8 GHz), 29 August 1994 (4.8 and $8.4 \mathrm{GHz}), 31$ October $1994(8.4 \mathrm{GHz}), 23$ December 1994 (8.4 GHz), and 7 April $1996(8.4 \mathrm{GHz})$. The VLA data had $50 \mathrm{MHz}$ of bandwidth in two sidebands in right (RCP) and left (LCP) circular polarization modes.

Data reduction was performed with the Astronomical Image Processing System (AIPS). 3C 48 was used as primary amplitude calibrator. Then amplitude and phase self-calibration was performed on $0954+658$. This forces $0954+658$ to have zero circular polarization. The amplitude calibration was transfered to M 81* and SN1993J before we performed phase selfcalibration on M 81* and SN1993J. Finally, we mapped all three sources in Stokes $I$ and $V$. Flux densities were determined by fitting an elliptical Gaussian component to the sources.

\subsection{New VLA observations}

In addition to the archival VLA data, we used the VLA to observe M 81* on 02 March 2001 and 9 dates between 15 June and 30 August 2001. During the latter period, the minimum and maximum separations between observing dates were 2 and 15 days, respectively. The VLA was in B configuration for the first observation and $\mathrm{C}$ array for the remaining observations. Observations were made at 5.0,8.4, 15 and $22 \mathrm{GHz}$ with $50 \mathrm{MHz}$ of bandwidth in two sidebands in RCP and LCP modes.

Each observation was between two and four hours in duration. Observing and analysis was performed with AIPS and followed the procedures outlined in Brunthaler et al. (2001). The extragalactic point source $\mathrm{J} 1044+719$ was used as a phase, amplitude and polarization calibrator as well as reference pointing source. The extragalactic point source $\mathrm{J} 1053+704$ was used to check for polarization calibration errors. 3C 286 was used as an absolute amplitude calibration source. Finally, we mapped all three sources in Stokes $I, U, Q$, and $V$. Flux densities were determined by fitting an elliptical Gaussian component to the sources.

We also used the VLA to observe M 81* on 09 August 2003 at $15 \mathrm{GHz}, 22 \mathrm{GHz}$, and $43 \mathrm{GHz}$ in polarimetric mode. The VLA was in A configuration with a resolution of about 50 milliarcsec at $43 \mathrm{GHz}$. The total integration time on $\mathrm{M} 81 *$ was 8,48 , and $52 \mathrm{~min}$ at 15,22 and $43 \mathrm{GHz}$ respectively and spread over a time range of $11 \mathrm{~h}$. Phase and amplitude calibration were performed using the nearby compact source, J1056+714. Phase self-calibration was also performed on M $81 *$ to eliminate the effects of atmospheric decorrelation. Polarization leakage calibration was performed using simultaneous full track observations of $\mathrm{J} 1056+714$ and $\mathrm{J} 1048+701$.

\subsection{BIMA observations}

We used the Berkeley-Illinois-Maryland Association (BIMA) array to observe M 81* at $3 \mathrm{~mm}$ wavelength (Welch et al. 1996). Observations were made in the multiplexed polarimetric mode described in Bower et al. (1999b). The receivers were tuned to sky frequencies of $82.8 \mathrm{GHz}$ (lower sideband) and $86.2 \mathrm{GHz}$ (upper sideband). The compact source $0954+658$ was used for phase calibration. Leakage calibration was determined from observations of 3C 279 on 11 November 2003. Observations of M 81* were made on 6 dates in September and October 2003 (Table A.4). BIMA observations at $230 \mathrm{GHz}$ on M $81 *$ were also performed in November 2003 (Table A.4). These data were also obtained in polarimetric mode with similar observing parameters to the $3 \mathrm{~mm}$ observations. Each observation was a $8 \mathrm{~h}$ track.

\subsection{Error analysis}

The Stokes parameter $V$ is measured as the difference between the left- and right-handed parallel polarization correlated 

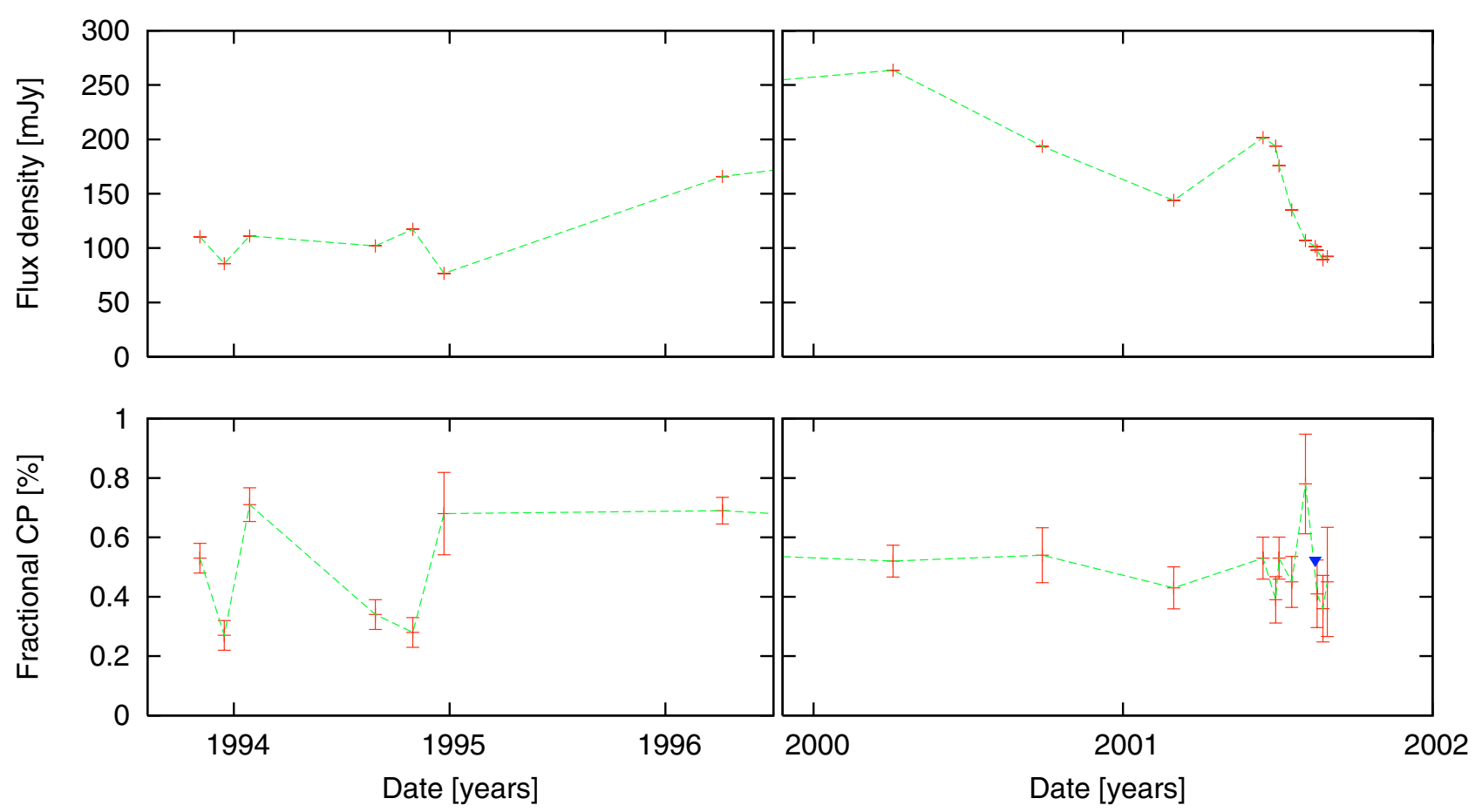

Fig. 2. Light-curve in total intensity (top) and fractional circular polarization of $\mathrm{M} 81 *$ at $8.4 \mathrm{GHz}$ for the archival data (left) and the new observations (right). The filled triangle is an $3 \sigma$ upper limit. The two data points in 2000 are taken from Brunthaler et al. (2001).

visibilities. Errors in circular polarization measurements with the VLA have numerous origins: thermal noise, gain errors, beam squint, second-order leakage corrections, unknown calibrator polarization, background noise and radio frequency interference. The errors caused by amplitude calibration errors, beam squint, and polarization leakage scale with the source strength, and therefore the fractional circular polarization is a more relevant indicator for the detection of circular polarization. A detailed discussion of these errors is given in Bower et al. (1999c) and Bower et al. (2002b). We calculated the systematic errors based on the model for the VLA for circular polarization from Bower et al. (2002b). For M 81*, SN1993J, and J1053+704 the errors on the fractional circular polarization in Tables A.1-A.3 are separated into statistical and systematic terms, while for the calibrator sources $0954+658$ and J1044+719 only the statistical error is given. The calibrator sources do not have a systematic error, since their circular polarization was assumed to be zero during the calibration.

\section{Results}

\subsection{Circular and linear polarization}

The results for the archival data at $4.8,8.4$, and $15 \mathrm{GHz}$ are shown in Tables A.1-A.3 respectively. We consider circular polarization as detected if the measured flux density exceeds the combined statistical and systematic errors (added in quadrature) by a factor of three. $0954+658$ showed no circular polarization as expected. M 81* showed circular polarization in all observations except one (16 December 1993 at $15 \mathrm{GHz}$ ). SN1993J showed circular polarization only in one epoch (29 August 1994 at $4.8 \mathrm{GHz}$ ) and no circular polarization in all other observations. The upper limits on the circular polarization of SN1993J are not very meaningful at $15 \mathrm{GHz}$ due to the low flux density and unexpected high noise.
The results for the new observations at $4.8,8.4$, and $15 \mathrm{GHz}$ are shown in Tables A.1-A.3 respectively. The $22 \mathrm{GHz}$ data and the high frequency observations on 9 August 2003 gave no useful limits on the circular polarization, mainly because the short integration time and higher systematic errors. At 4.8. 8.4, and $15 \mathrm{GHz}, 1044+719$ showed no circular polarization as expected. M 81* showed circular polarization in three epochs at $4.8 \mathrm{GHz}$, all except one epoch at $8.4 \mathrm{GHz}$ and four epochs at $15 \mathrm{GHz}$. The check source $1053+704$ showed only circular polarization in two epochs at $15 \mathrm{GHz}$.

The three detections of circular polarization in the check sources SN1993J and 1053+704 are caused by either remaining amplitude calibration errors or by a small level of circular polarization in the sources. Since both sources show no circular polarization at $8.4 \mathrm{GHz}$, the frequency band with the highest sensitivity, it is most likely that the detected circular polarization in SN1993J and 1053+704 comes from residual amplitude calibration errors. The fractional circular polarization in $\mathrm{M} 81$ * at $15 \mathrm{GHz}$ is higher by a factor of 2 and 4 than in $1053+704$ in the observations on 15 June 2001 and 4 July 2001 respectively. In these two cases, the measured circular polarization of $\mathrm{M} 81^{*}$ is probably only partly caused by the amplitude calibration errors.

In the BIMA observations at 86 and $230 \mathrm{GHz}$ neither linear nor circular polarization is detected for $\mathrm{M} 81 *$ in any individual epoch. Mean linear polarization at $86 \mathrm{GHz}$ is $1.2 \mathrm{mJy}$, or $1.6 \%$ $(3 \sigma)$. Mean circular polarization at $86 \mathrm{GHz}$ is $2.8 \pm 0.4 \mathrm{mJy}$, or $3.9 \pm 0.5 \%$. Although this is formally a detection, it is not clear whether systematic errors are significant. Due to the low flux density, limits on the polarized flux density are not significant at $230 \mathrm{GHz}$.

The $8.4 \mathrm{GHz}$ data seems to be the most reliable data since M 81* showed circular polarization in all epochs except one while the check sources SN1993J and 1053+704 never showed circular polarization. The light-curve of total intensity and fractional circular polarization is shown in Fig. 2. The fractional circular polarization shows significant variability on timescales of 

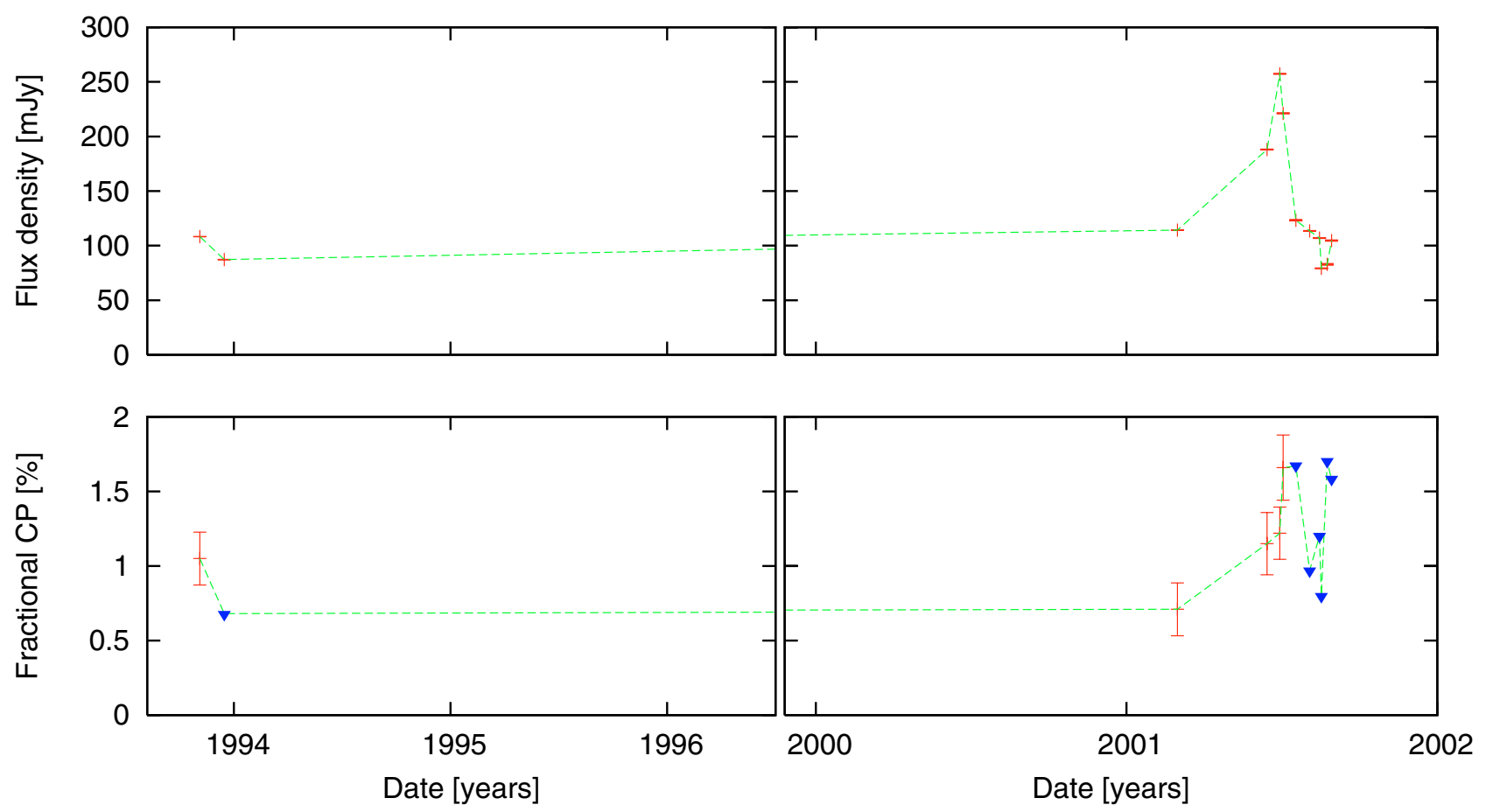

Fig. 3. Light-curve in total intensity (top) and fractional circular polarization of M $81^{*}$ at $15 \mathrm{GHz}$ for the archival data (left) and the new observations (right). The filled triangles are $3 \sigma$ upper limits.

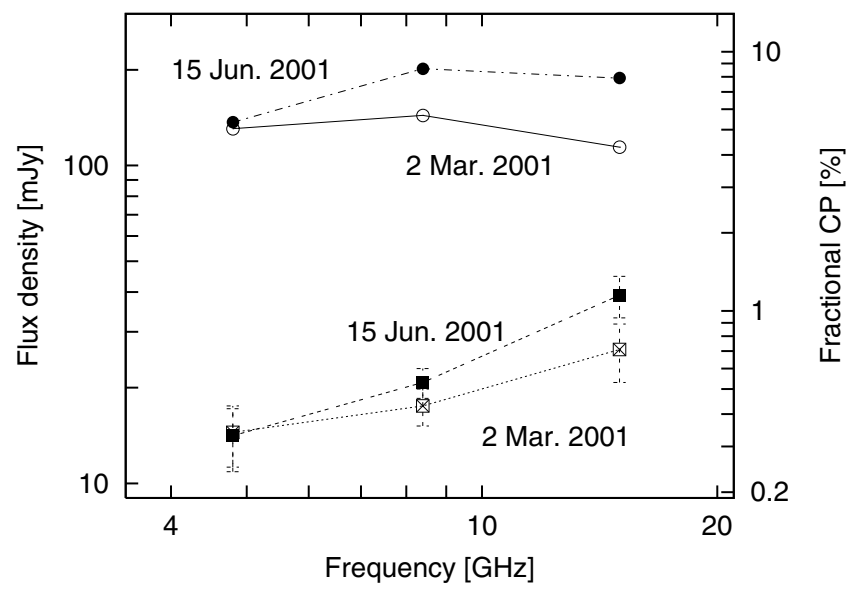

Fig. 4. Spectra of the total intensity (circles) and the fractional circular polarization (squares) from 2 March 2001 (open) and 15 June 2001 (filled).

a few weeks which is not correlated with the variability in the total intensity. Between 4 August 2001 and 16 August 2001, the fractional circular polarization at $8.4 \mathrm{GHz}$ dropped from $0.78 \%$ to less than $0.35 \%$ while the total intensity showed no significant change. However it is remarkable that, despite the strong variability, the sign of the circular polarization is always positive. At the other two frequencies, the sign is also positive when circular polarization is detected in $\mathrm{M} 81^{*}$.

The spectrum of the fractional circular polarization is inverted $\left(\alpha>0\right.$ for $\left.m_{\mathrm{c}} \propto v^{\alpha}\right)$ with values between 0.4 and 2 in most epochs when it was detected at more than one frequency. The mean spectral index between 4.8 and $8.4 \mathrm{GHz}$ is 0.51 , while the mean spectral index between 8.4 and $15 \mathrm{GHz}$ is 1.52 . Only the observation on 18 August 2001 shows a steep spectrum of $\alpha=-0.77$ between 4.8 and $8.4 \mathrm{GHz}$.

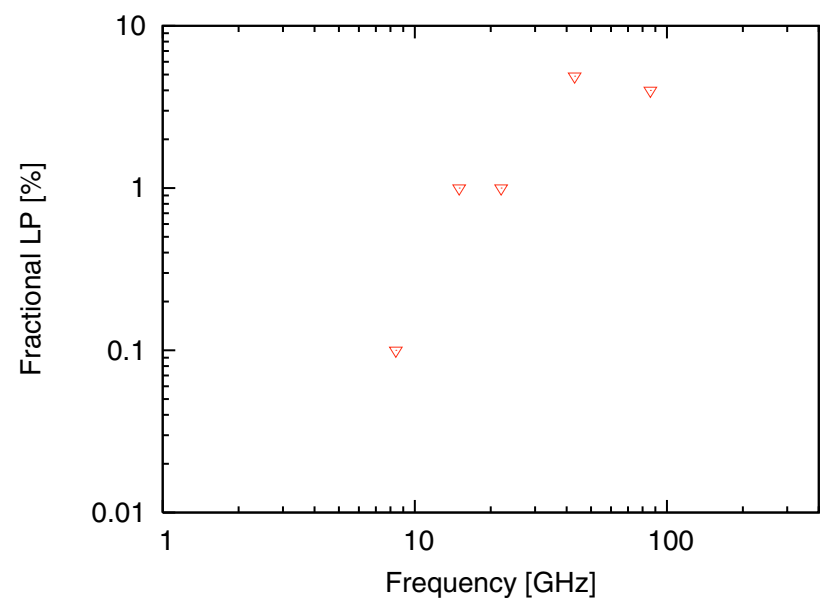

Fig. 5. $3 \sigma$ upper limits on the linear polarization of $\mathrm{M} 81 *$ up to $86 \mathrm{GHz}$. The $8.4 \mathrm{GHz}$ data point is taken from Bower et al. (2002a).

Linear polarization was not detected in the VLA observations on 9 August 2003 and the BIMA observations at $86 \mathrm{GHz}$ (Fig. 5). The archival data was not searched for linear polarization.

\subsection{Total intensity}

The high frequency VLA data taken on 9 August 2003 give a simultaneous total intensity spectrum of $\mathrm{M} 81 *$ that shows the flux density decreasing from 15 to $43 \mathrm{GHz}$ with a spectral index of $\sim-0.6$. In the BIMA observations M $81 *$ is clearly detected at $86 \mathrm{GHz}$ in total intensity and is strongly variable on a time scale $\sim 10$ days. The mean total intensity is $71 \mathrm{mJy}$. Due to low sensitivity, M $81 *$ is only marginally detected at $230 \mathrm{GHz}$ in total intensity. The mean flux density for M $81 *$ from this observation is $31 \pm 4 \pm 15 \mathrm{mJy}$, where the first error is the statistical error 


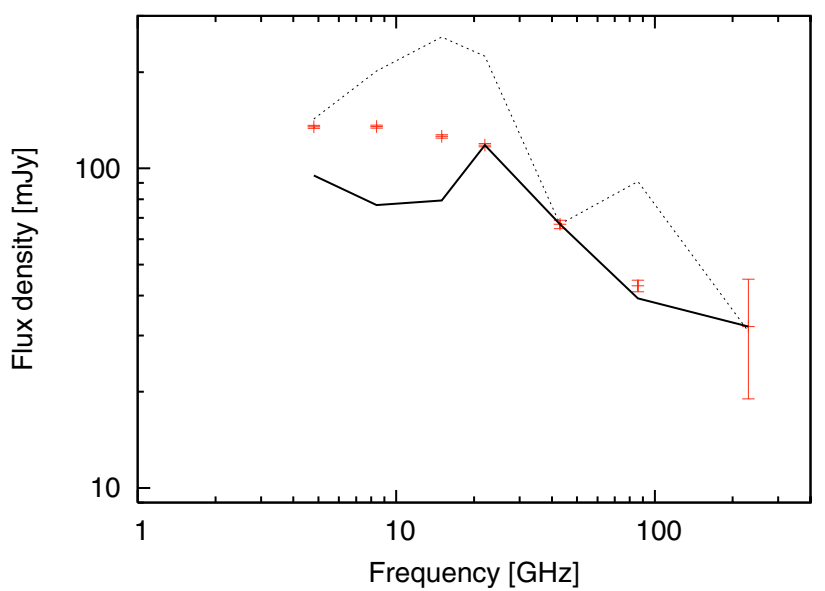

Fig. 6. Non simultaneous broad-band spectrum of M 81*. Also shown are the maximal (dashed line) and minimal (solid line) measured values at each frequency.

and the second error is the systematic error due to decorrelation. Atmospheric decorrelation may be serious and these results may significantly underestimate the total flux density of M 81* (Table A.4).

Figure 6 shows a non simultaneous broad-band spectrum of M 81*. The $15-43 \mathrm{GHz}$ data points are from the VLA observations on 9 August 2003. The 4.8 and $8.4 \mathrm{GHz}$ data points are from the observation on 19 July 2001, where the $15 \mathrm{GHz}$ flux was comparable to the $15 \mathrm{GHz}$ flux in the 9 August 2003 observation. The $86 \mathrm{GHz}$ and $230 \mathrm{GHz}$ data points are from the BIMA observations on 7 September 2003 and November 2003 respectively. Also shown are the maximal and minimal measured values at each frequency in our observations. Although the spectrum is not simultaneous it is clear that the spectrum declines towards higher frequencies.

M 81* underwent a flare in total intensity during the new VLA observations between June and August 2001. The peak was reached at $8.4 \mathrm{GHz}$ before 15 June 2001 , while the flux density continued to rise at 4.8 until 4 July 2001 . At $15 \mathrm{GHz}$, the peak was reached in 30 June 2001. Figure 7 shows the spectral indices between 4.8 and $8.4 \mathrm{GHz}$, and bewteen 8.4 and $15 \mathrm{GHz}$ during this flare. The spectrum at the lower frequencies shows a smooth transition from an inverted $(\alpha \sim+0.7)$ to a steep $(\alpha \sim$ -0.4 ) spectrum. At higher frequencies, the spectral index does not follow a trend and is scattered between -0.4 and +0.5 . The fast change in spectral index between 4.8 and $8.4 \mathrm{GHz}$ could be caused by a drop in the turnover frequency of a syncrotron selfabsorpted jet from above 8.4 to below $4.8 \mathrm{GHz}$ and should be accompanied by a fast expansion of the jet. This behaviour is known in other active galactic nuclei (e.g. III $\mathrm{Zw} 2$ : Brunthaler et al. 2000; Brunthaler et al. 2005). The scatter of the spectral index between 8.4 and $15 \mathrm{GHz}$ could be caused by multiple subflares that occur at $15 \mathrm{GHz}$.

\section{Discussion}

The origin of circular polarization in AGN is still not known. Several mechanisms have been proposed in the literature. Interstellar propagation effects predict a very steep spectrum (Macquart \& Melrose 2000) which is not consistent with our observations. One possible mechanism could be Faraday conversion (Pacholczyk 1977; Jones \& Odell 1977) of linear polarization to circular polarization caused by the lowest energy

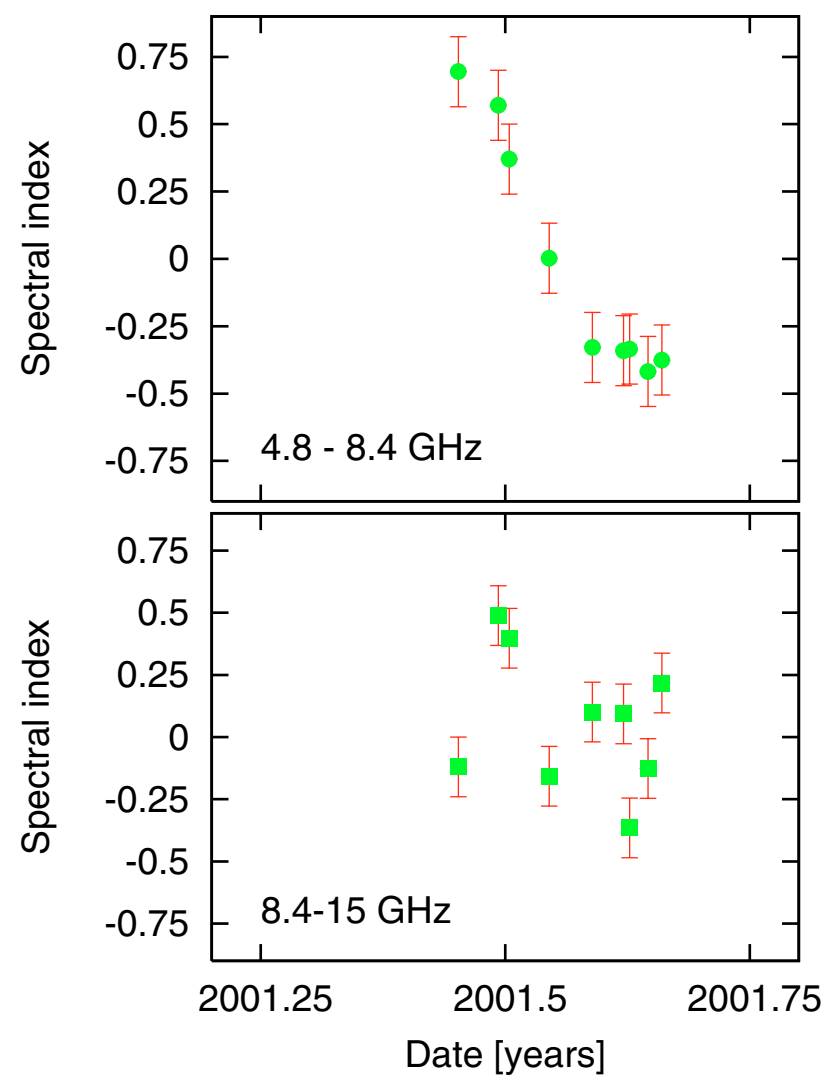

Fig. 7. Spectral indices of $\mathrm{M} 81 *$ between 4.8 and $8.4 \mathrm{GHz}$ (top) and 8.4 and $15 \mathrm{GHz}$ (bottom) during the flare in the new VLA observations.

relativistic electrons. Bower et al. (1999c) proposed a simple model for Sgr A* in which low-energy electrons reduce linear polarization through Faraday de-polarization and convert linear polarization into circular polarization. Faraday conversion can also affect the spectral properties of circular polarization and may lead to a variety of spectral indices, including inverted spectra (Jones \& Odell 1977). In inhomogeneous sources, conversion can produce relatively high fractional circular polarization (Jones 1988). Gyro-synchrotron emission, can also lead to high circular polarization with an inverted spectrum and low linear polarization (Ramaty 1969). However, this mechanism is to some degree related and also requires that $\mathrm{M} 81^{*}$ and $\mathrm{Sgr} \mathrm{A}^{*}$ both contain a rather large number of low-energy electrons. Faraday conversion is also favored by Beckert \& Falcke (2002) and Ruszkowski \& Begelman (2002).

The long-term stability of the sign of circular polarization suggests that the Faraday conversion is connected to fundamental properties of the source and the material which is responsible for the conversion. It requires uniformity in the magnetic pole and accretion conditions over the observed timescales. One possible scenario is described by Enßlin (2003) where the sign of the circular polarization is connected to the sense of rotation of the central engine. In this scenario M $81^{*}$ is expected to rotate counter-clockwise.

The size of the radio emission of $\mathrm{M} 81^{*}$ at $8.4 \mathrm{GHz}$ is $\sim 0.45$ mas, or $\sim 1800 \mathrm{AU}$ at $4 \mathrm{Mpc}$ (Bietenholz et al. 1996). The fact that $\mathrm{M} 81 *$ is depolarized at at level of $<0.1 \%$ at the same frequency requires that the depolarizing material is also present at a scale of $\sim 1800 \mathrm{AU}$.

While the polarization properties of $\mathrm{M} 81^{*}$ and $\operatorname{Sgr} \mathrm{A}^{*}$ are strikingly similar the total intensity spectrum seems to be 
different. In Sgr A*, the radio flux density rises towards the submm regime (e.g. Zylka et al. 1995; Falcke \& Markoff 2000). Our measurements indicate a different trend for M 81*. Although the spectrum in Fig. 6 is not simultaneous and M 81* shows strong variability our measured flux densities at 86 and $230 \mathrm{GHz}$ are lower than the typical flux densities at centimeter wavelengths. This is different from the results in Reuter \& Lesch (1996) who find an inverted spectrum up to $\sim 100 \mathrm{GHz}$. We can not tell whether we observed $\mathrm{M} 81^{*}$ in a phase with unusual low millimeter emission or the Reuter \& Lesch (1996) observations were made during an outburst at millimeter wavelengths. A simultaneous monitoring project from centimeter to millimeter wavelengths would be needed to decide this question.

Linear polarization is not detected for M $81^{*}$ at any wavelength longward of $3.6 \mathrm{~mm}$. The presence of a jet at VLBI resolution, radio synchrotron emission, and circular polarization suggest that $\mathrm{M} 81^{*}$ is intrinsically linearly polarized but depolarized during propagation through a magnetized plasma. The case is similar to that of Sgr A*, which is detected in linear polarization only at wavelengths shortward of $3.6 \mathrm{~mm}$. For Sgr A*, the detection of linear polarization at short wavelengths provides an upper limit to the rotation measure of a few times $10^{6} \mathrm{rad} \mathrm{m}^{-2}$. This provides an upper limit to the accretion rate of $\sim 10^{-7} M_{\odot} \mathrm{y}^{-1}$. For M $81^{*}$, we find a lower limit to the rotation measure of $\sim 10^{4} \mathrm{rad} \mathrm{m}^{-2}$ for the case of beam depolarization and $\sim 4 \times 10^{5} \mathrm{rad} \mathrm{m}^{-2}$ for the case of bandwidth depolarization, under the assumption that the intrinsic source is polarized. The lesser value could originate in the dense interstellar medium but the larger value exceeds that seen anywhere in the ISM. ADAF and Bondi-Hoyle accretion models will depolarize the source unless the accretion rate falls below $10^{-9} M_{\odot} \mathrm{y}^{-1}$ (Quataert \& Gruzinov 2000). However, for radiatively inefficient accretion flows, the larger of the RM limits implies a lower limit to the accretion rate of $10^{-7} M_{\odot} \mathrm{y}^{-1}$. The accretion rate necessary for the X-ray luminosity is $10^{-5} M_{\odot} \mathrm{y}^{-1}$. Since bandwidth depolarization effects decrease as $\lambda^{3}$, measurement of the linear polarization at a wavelength of $0.8 \mathrm{~mm}$ would increase the accuracy of the accretion rate constraint by nearly two orders of magnitude.

\section{Summary and conclusion}

We have presented VLA observations of M 81* from 1994 until 2002 that show that circular polarization is present at 4.8, 8.4, and $15 \mathrm{GHz}$ in absence of linear polarization. The fractional circular polarization is variable on timescales of days and months and not correlated with the total flux density of the source. The sign of the circular polarization was, if detected, at all frequencies and times always positive. The polarization properties are strikingly similar to the properties of Sgr A*, the central radio source in the Milky Way. This supports the hypothesis that M $81 *$ is a scaled up version of Sgr A*. Aitken et al. (2000) and Bower et al. (2003) detected linear polarization at $230 \mathrm{GHz}$ and higher frequencies that also shows variability (Bower et al. 2005; Marrone et al. 2006). Given the similarity between M 81* and $\mathrm{Sgr} \mathrm{A}^{*}$ we expect to see also linear polarization in $\mathrm{M} 81^{*}$ at higher frequencies.

Acknowledgements. This research partially supported by the DFG Priority Programme 1177. The National Radio Astronomy Observatory is a facility of the National Science Foundation operated under cooperative agreement by Associated Universities, Inc.

\section{References}

Aitken, D. K., Greaves, J., Chrysostomou, A., et al. 2000, ApJ, 534, L173 Bartel, N., Bietenholz, M. F., Rupen, M. P., et al. 1994, Nature, 368, 610 Beckert, T., Falcke, H. 2002, A\&A, 388, 1106

Bietenholz, M. F., Bartel, N., \& Rupen, M. P. 2000, ApJ, 532, 895

Bietenholz, M. F., Bartel, N., \& Rupen, M. P. 2004, ApJ, 615, 173

Bietenholz, M. F., Bartel, N., Rupen, M. P., et al. 1996, ApJ, 457, 604

Bower, G. C., Backer, D. C., Zhao, J., Goss, M., \& Falcke, H. 1999a, ApJ, 521, 582 Bower, G. C., Wright, M. C. H., Backer, D. C., \& Falcke, H. 1999b, ApJ, 527, 851 Bower, G. C., Falcke, H., \& Backer, D. C. 1999c, ApJ, 523, L29

Bower, G. C., Falcke, H., \& Mellon, R. R. 2002a, ApJ, 578, L103 Bower, G. C., Falcke, H., Sault, R. J., \& Backer, D. C. 2002b, ApJ, 571, 843 Bower, G. C., Wright, M. C. H., Falcke, H., \& Backer, D. C. 2003, ApJ, 588, 331 Bower, G. C., Falcke, H., Herrnstein, R. M., et al. 2004, Science, 304, 704 Bower, G. C., Falcke, H., Wright, M. C., \& Backer, D. C. 2005, ApJ, 618, L29 Brunthaler, A., Falcke, H., Bower, G. C., et al. 2000, A\&A, 357, L45 Brunthaler, A., Bower, G. C., Falcke, H., \& Mellon, R. R. 2001, ApJ, 560, L123 Brunthaler, A., Falcke, H., Bower, G. C., et al. 2005, A\&A, 435, 497 Enßlin, T. A. 2003, A\&A, 401, 499

Falcke, H. 1996, ApJ, 464, L67

Falcke, H., \& Markoff, S. 2000, A\&A, 362, 113

Ho, L. C., Filippenko, A. V., \& Sargent, W. L. W. 1996, ApJ, 462, 183

Jones, T. W. 1988, ApJ, 332, 678

Jones, T. W., \& Odell, S. L. 1977, ApJ, 214, 522

Macquart, J.-P., \& Melrose, D. B. 2000, ApJ, 545, 798

Marrone, D. P., Moran, J. M., Zhao, J., \& Rao, R. 2006, ApJ, 640, 308

Melia, F., \& Falcke, H. 2001, ARA\&A, 39, 309

Pacholczyk, A. G. 1977, Oxford Pergamon Press International Series on Natural Philosophy, 89

Quataert, E., \& Gruzinov, A. 2000, ApJ, 545, 842

Ramaty, R. 1969, ApJ, 158, 753

Rayner, D. P., Norris, R. P., \& Sault, R. J. 2000, MNRAS, 319, 484

Reuter, H.-P., \& Lesch, H. 1996, A\&A, 310, L5

Ruszkowski, M., \& Begelman, M. C. 2002, ApJ, 573, 485

Shen, Z.-Q., Lo, K. Y., Liang, M.-C., Ho, P. T. P., \& Zhao, J.-H. 2005, Nature, 438, 62 Wardle, J. F. C., Homan, D. C., Ojha, R., \& Roberts, D. H. 1998, Nature, 395, 457

Welch, W. J., Thornton, D. D., Plambeck, R. L., et al. 1996, PASP, 108, 93

Zylka, R., Mezger, P. G., Ward-Thompson, D., Duschl, W. J., \& Lesch, H. 1995, A\&A, 297, 83 
A. Brunthaler et al.: Radio linear and circular polarization from M 81*, Online Material p 1

\section{Online Material}


A. Brunthaler et al.: Radio linear and circular polarization from M 81*, Online Material p 2

Appendix A: Tables

Table A.1. Circularly polarized flux at $4.8 \mathrm{GHz}$ for $\mathrm{M} 81 *$ and calibrators. The errors on the fractional circular polarization are separated into statistical and systematic terms for the target and check source. For the calibrator source only the statistical error is given.

\begin{tabular}{|c|c|c|c|c|c|}
\hline Date & Source & $\begin{array}{r}I \\
{[\mathrm{mJy}]}\end{array}$ & $\begin{array}{r}P_{\mathrm{c}} \\
{[\mathrm{mJy}]}\end{array}$ & $\begin{array}{r}\mathrm{rms} \\
{[\mathrm{mJy}]}\end{array}$ & $\begin{array}{r}m_{\mathrm{c}} \\
{[\%]}\end{array}$ \\
\hline \multirow[t]{3}{*}{28 Jan. 1994} & $0954+658$ & 622.2 & $<0.43$ & 0.11 & $<0.07 \pm 0.02$ \\
\hline & M $81 *$ & 95.0 & 0.36 & 0.06 & $0.38 \pm 0.06 \pm 0.04$ \\
\hline & SN1993J & 77.1 & $<0.17$ & 0.05 & $<0.22 \pm 0.06 \pm 0.03$ \\
\hline \multirow[t]{3}{*}{21 Apr. 1994} & $0954+658$ & 534.7 & $<0.21$ & 0.07 & $<0.04 \pm 0.01$ \\
\hline & M $81 *$ & 114.1 & 0.52 & 0.05 & $0.46 \pm 0.04 \pm 0.03$ \\
\hline & SN1993J & 62.1 & $<0.22$ & 0.04 & $<0.35 \pm 0.06 \pm 0.03$ \\
\hline \multirow[t]{3}{*}{29 Aug. 1994} & $0954+658$ & 597.2 & $<0.18$ & 0.06 & $<0.03 \pm 0.01$ \\
\hline & M $81 *$ & 109.0 & 0.21 & 0.04 & $0.19 \pm 0.04 \pm 0.03$ \\
\hline & SN1993J & 54.6 & 0.18 & 0.03 & $0.33 \pm 0.05 \pm 0.03$ \\
\hline \multirow[t]{3}{*}{02 Mar. 2001} & $1044+719$ & 1568.2 & 0.11 & 0.11 & $<0.01 \pm 0.01$ \\
\hline & M $81 *$ & 130.5 & 0.44 & 0.10 & $0.34 \pm 0.08 \pm 0.05$ \\
\hline & $1053+704$ & 388.2 & 0.03 & 0.07 & $<0.01 \pm 0.02 \pm 0.06$ \\
\hline \multirow[t]{3}{*}{15 Jun. 2001} & $1044+719$ & 1710.9 & 0.33 & 0.35 & $<0.02 \pm 0.02$ \\
\hline & M $81 *$ & 136.7 & 0.45 & 0.11 & $0.33 \pm 0.08 \pm 0.05$ \\
\hline & $1053+704$ & 490.1 & -0.58 & 0.21 & $<0.12 \pm 0.04 \pm 0.06$ \\
\hline \multirow[t]{3}{*}{30 Jun. 2001} & $1044+719$ & 1645.4 & 0.4 & 0.32 & $<0.02 \pm 0.02$ \\
\hline & M $81 *$ & 140.9 & 0.3 & 0.12 & $<0.21 \pm 0.09 \pm 0.05$ \\
\hline & $1053+704$ & 497.5 & -1.41 & 0.33 & $<0.28 \pm 0.07 \pm 0.07$ \\
\hline \multirow[t]{3}{*}{04 Jul. 2001} & $1044+719$ & 1687.5 & 0.01 & 0.01 & $<0.01 \pm 0.01$ \\
\hline & M $81 *$ & 142.8 & 0.12 & 0.05 & $<0.09 \pm 0.04 \pm 0.04$ \\
\hline & $1053+704$ & 484 & 0.01 & 0.01 & $<0.01 \pm 0.01 \pm 0.05$ \\
\hline \multirow[t]{3}{*}{19 Jul. 2001} & $1044+719$ & 1645.3 & 0.08 & 0.24 & $<0.01 \pm 0.05$ \\
\hline & M $81 *$ & 134.9 & 0.46 & 0.19 & $<0.34 \pm 0.14 \pm 0.07$ \\
\hline & $1053+704$ & 516.8 & 0.12 & 0.12 & $<0.02 \pm 0.02 \pm 0.10$ \\
\hline \multirow[t]{3}{*}{04 Aug. 2001} & $1044+719$ & 1641.8 & 0.1 & 0.31 & $<0.01 \pm 0.02$ \\
\hline & M 81* & 128.6 & 0.07 & 0.07 & $<0.05 \pm 0.05 \pm 0.07$ \\
\hline & $1053+704$ & 564.3 & 0.14 & 0.14 & $<0.02 \pm 0.02 \pm 0.10$ \\
\hline \multirow[t]{3}{*}{16 Aug. 2001} & $1044+719$ & 1636.5 & 0.06 & 0.23 & $<0.01 \pm 0.01$ \\
\hline & M $81 *$ & 122.7 & 0.44 & 0.18 & $<0.36 \pm 0.15 \pm 0.07$ \\
\hline & $1053+704$ & 524.3 & -0.38 & 0.23 & $<0.07 \pm 0.04 \pm 0.10$ \\
\hline \multirow[t]{3}{*}{18 Aug. 2001} & $1044+719$ & 1607.5 & 0.01 & 0.01 & $<0.01 \pm 0.01$ \\
\hline & M $81 *$ & 118.2 & 0.74 & 0.16 & $0.63 \pm 0.14 \pm 0.07$ \\
\hline & $1053+704$ & 532.6 & 0.14 & 0.14 & $<0.03 \pm 0.03 \pm 0.10$ \\
\hline \multirow[t]{3}{*}{25 Aug. 2001} & $1044+719$ & 1602.7 & 0.02 & 0.15 & $<0.01 \pm 0.01$ \\
\hline & M $81 *$ & 112.7 & 0.37 & 0.16 & $<0.33 \pm 0.14 \pm 0.07$ \\
\hline & $1053+704$ & 546 & 0.13 & 0.13 & $<0.02 \pm 0.02 \pm 0.10$ \\
\hline \multirow[t]{3}{*}{30 Aug. 2001} & $1044+719$ & 1655.7 & 0.13 & 0.13 & $<0.01 \pm 0.01$ \\
\hline & M $81 *$ & 113.9 & 0.23 & 0.11 & $<0.2 \pm 0.10 \pm 0.07$ \\
\hline & $1053+704$ & 548 & -0.08 & 0.14 & $<0.01 \pm 0.03 \pm 0.10$ \\
\hline
\end{tabular}


A. Brunthaler et al.: Radio linear and circular polarization from M 81*, Online Material p 3

Table A.2. Circularly polarized flux at $8.4 \mathrm{GHz}$ for $\mathrm{M} 81 *$ and calibrators. The errors on the fractional circular polarization are separated into statistical and systematic terms for the target and check source. For the calibrator source only the statistical error is given.

\begin{tabular}{|c|c|c|c|c|c|}
\hline Date & Source & $\begin{array}{r}I \\
{[\mathrm{mJy}]}\end{array}$ & $\begin{array}{r}P_{\mathrm{c}} \\
{[\mathrm{mJy}]}\end{array}$ & $\begin{array}{r}\mathrm{rms} \\
{[\mathrm{mJy}]}\end{array}$ & $\begin{array}{r}m_{\mathrm{c}} \\
{[\%]}\end{array}$ \\
\hline \multirow[t]{3}{*}{05 Nov. 1993} & $0954+658$ & 663.5 & $<0.27$ & 0.04 & $<0.04 \pm 0.01$ \\
\hline & M 81* & 110.4 & 0.59 & 0.03 & $0.53 \pm 0.03 \pm 0.04$ \\
\hline & SN1993J & 62.2 & $<0.07$ & 0.02 & $<0.11 \pm 0.03 \pm 0.03$ \\
\hline \multirow[t]{3}{*}{16 Dec. 1993} & $0954+658$ & 655.5 & $<0.12$ & 0.04 & $<0.02 \pm 0.01$ \\
\hline & M $81 *$ & 85.7 & 0.23 & 0.03 & $0.27 \pm 0.04 \pm 0.03$ \\
\hline & SN1993J & 54.7 & $<0.06$ & 0.02 & $<0.11 \pm 0.04 \pm 0.03$ \\
\hline \multirow[t]{3}{*}{28 Jan. 1994} & $0954+658$ & 600.6 & $<0.24$ & 0.08 & $<0.04 \pm 0.01$ \\
\hline & M $81 *$ & 111.2 & 0.79 & 0.04 & $0.71 \pm 0.04 \pm 0.04$ \\
\hline & SN1993J & 48.9 & $<0.14$ & 0.03 & $<0.29 \pm 0.06 \pm 0.03$ \\
\hline \multirow[t]{3}{*}{29 Aug. 1994} & $0954+658$ & 648.1 & $<0.11$ & 0.04 & $<0.02 \pm 0.01$ \\
\hline & M 81* & 102.0 & 0.35 & 0.03 & $0.34 \pm 0.03 \pm 0.04$ \\
\hline & SN1993J & 34.5 & $<0.08$ & 0.03 & $<0.23 \pm 0.09 \pm 0.03$ \\
\hline \multirow[t]{3}{*}{31 Oct. 1994} & $0954+658$ & 670.9 & $<0.12$ & 0.04 & $<0.02 \pm 0.01$ \\
\hline & M $81 *$ & 117.2 & 0.33 & 0.03 & $0.28 \pm 0.03 \pm 0.04$ \\
\hline & SN1993J & 33.5 & $<0.08$ & 0.03 & $<0.24 \pm 0.09 \pm 0.03$ \\
\hline \multirow[t]{3}{*}{23 Dec. 1994} & $0954+658$ & 693.7 & $<0.43$ & 0.14 & $<0.06 \pm 0.02$ \\
\hline & M $81 *$ & 76.7 & 0.52 & 0.09 & $0.68 \pm 0.12 \pm 0.07$ \\
\hline & SN1993J & 30.4 & $<0.22$ & 0.07 & $<0.72 \pm 0.23 \pm 0.05$ \\
\hline \multirow[t]{3}{*}{07 Apr. 1996} & $0954+658$ & 786.3 & $<0.11$ & 0.04 & $<0.01 \pm 0.01$ \\
\hline & M $81 *$ & 165.8 & 1.15 & 0.03 & $0.69 \pm 0.02 \pm 0.04$ \\
\hline & SN1993J & 20.5 & $<0.15$ & 0.05 & $<0.73 \pm 0.24 \pm 0.04$ \\
\hline \multirow{3}{*}{02 Mar. 2001} & $1044+719$ & 1478.7 & 0.13 & 0.13 & $<<0.01 \pm 0.01$ \\
\hline & M $81 *$ & 143.8 & 0.62 & 0.07 & $0.43 \pm 0.05 \pm 0.05$ \\
\hline & $1053+704$ & 524.8 & 0.35 & 0.10 & $<0.07 \pm 0.02 \pm 0.06$ \\
\hline \multirow[t]{3}{*}{15 Jun. 2001} & $1044+719$ & 2136.1 & 0.11 & 0.11 & $<0.01 \pm 0.01$ \\
\hline & M $81 *$ & 201.7 & 1.08 & 0.10 & $0.53 \pm 0.05 \pm 0.05$ \\
\hline & $1053+704$ & 1006.1 & 0.17 & 0.17 & $<0.02 \pm 0.02 \pm 0.06$ \\
\hline \multirow[t]{3}{*}{30 Jun. 2001} & $1044+719$ & 1488.6 & 0.01 & 0.01 & $<0.01 \pm 0.01$ \\
\hline & M $81 *$ & 193.9 & 0.75 & 0.11 & $0.39 \pm 0.06 \pm 0.05$ \\
\hline & $1053+704$ & 763.0 & 0.34 & 0.19 & $<0.04 \pm 0.02 \pm 0.07$ \\
\hline \multirow[t]{3}{*}{ 04 Jul. 2001} & $1044+719$ & 1525.8 & 0.57 & 0.57 & $<0.04 \pm 0.04$ \\
\hline & M $81 *$ & 175.7 & 0.93 & 0.09 & $0.53 \pm 0.05 \pm 0.05$ \\
\hline & $1053+704$ & 753.3 & 0.13 & 0.13 & $<0.02 \pm 0.02 \pm 0.06$ \\
\hline \multirow[t]{3}{*}{ 19 Jul. 2001} & $1044+719$ & 1474.8 & 0.10 & 0.10 & $<0.01 \pm 0.01$ \\
\hline & M $81 *$ & 135.1 & 0.60 & 0.09 & $0.45 \pm 0.07 \pm 0.05$ \\
\hline & $1053+704$ & 769.2 & 0.27 & 0.13 & $<0.04 \pm 0.02 \pm 0.07$ \\
\hline \multirow[t]{3}{*}{04 Aug. 2001} & $1044+719$ & 1485.4 & 0.12 & 0.12 & $<0.01 \pm 0.01$ \\
\hline & M $81 *$ & 107.0 & 0.84 & 0.17 & $0.78 \pm 0.16 \pm 0.05$ \\
\hline & $1053+704$ & 785.1 & 0.57 & 0.26 & $<0.07 \pm 0.03 \pm 0.07$ \\
\hline \multirow[t]{3}{*}{16 Aug. 2001} & $1044+719$ & 1469.1 & 0.27 & 0.27 & $<0.02 \pm 0.02$ \\
\hline & M $81 *$ & 101.4 & 0.36 & 0.16 & $<0.35 \pm 0.16 \pm 0.07$ \\
\hline & $1053+704$ & 758.3 & 1.20 & 0.27 & $<0.16 \pm 0.04 \pm 0.10$ \\
\hline \multirow[t]{3}{*}{18 Aug. 2001} & $1044+719$ & 1476.8 & 0.41 & 0.65 & $<0.03 \pm 0.03$ \\
\hline & M $81 *$ & 98.0 & 0.40 & 0.09 & $0.41 \pm 0.09 \pm 0.07$ \\
\hline & $1053+704$ & 775.2 & 0.41 & 0.30 & $<0.05 \pm 0.04 \pm 0.10$ \\
\hline \multirow[t]{3}{*}{25 Aug. 2001} & $1044+719$ & 1406.9 & 0.11 & 0.11 & $<0.01 \pm 0.01$ \\
\hline & M $81 *$ & 89.2 & 0.32 & 0.09 & $0.36 \pm 0.10 \pm 0.05$ \\
\hline & $1053+704$ & 746.0 & 1.30 & 0.24 & $<0.17 \pm 0.03 \pm 0.07$ \\
\hline \multirow[t]{3}{*}{30 Aug. 2001} & $1044+719$ & 1520 & 0.17 & 0.17 & $<0.01 \pm 0.01$ \\
\hline & M $81 *$ & 92.3 & 0.42 & 0.16 & $0.45 \pm 0.17 \pm 0.07$ \\
\hline & $1053+704$ & 789.2 & 0.30 & 0.30 & $<0.04 \pm 0.04 \pm 0.10$ \\
\hline
\end{tabular}


A. Brunthaler et al.: Radio linear and circular polarization from M 81*, Online Material p 4

Table A.3. Circularly polarized flux at $15 \mathrm{GHz}$ for $\mathrm{M} 81^{*}$ and calibrators. The errors on the fractional circular polarization are separated into statistical and systematic terms for the target and check source. For the calibrator source only the statistical error is given.

\begin{tabular}{|c|c|c|c|c|c|}
\hline Date & Source & $\begin{array}{r}I \\
{[\mathrm{mJy}]}\end{array}$ & $\begin{array}{r}P_{\mathrm{c}} \\
{[\mathrm{mJy}]}\end{array}$ & $\begin{array}{r}\mathrm{rms} \\
{[\mathrm{mJy}]}\end{array}$ & $\begin{array}{r}m_{\mathrm{c}} \\
{[\%]}\end{array}$ \\
\hline \multirow[t]{3}{*}{05 Nov. 1993} & $0954+658$ & 664.5 & $<0.26$ & 0.09 & $<0.04 \pm 0.01$ \\
\hline & M $81 *$ & 108.2 & 1.14 & 0.18 & $1.05 \pm 0.17 \pm 0.05$ \\
\hline & SN1993J & 42.3 & $<4.05$ & 1.35 & $<9.57 \pm 3.19 \pm 0.03$ \\
\hline \multirow[t]{3}{*}{16 Dec. 1993} & $0954+658$ & 606.4 & $<0.28$ & 0.09 & $<0.05 \pm 0.01$ \\
\hline & M $81 *$ & 87.2 & $<0.06$ & 0.19 & $<0.07 \pm 0.22 \pm 0.05$ \\
\hline & SN1993J & 39.0 & $<9.0$ & 3.32 & $<23.0 \pm 8.51 \pm 0.03$ \\
\hline \multirow[t]{3}{*}{02 Mar. 2001} & $1044+719$ & 999.8 & 0.03 & 0.12 & $<0.01 \pm 0.01$ \\
\hline & M $81 *$ & 114.2 & 0.81 & 0.19 & $0.71 \pm 0.17 \pm 0.05$ \\
\hline & $1053+704$ & 485.2 & 0.25 & 0.18 & $<0.05 \pm 0.04 \pm 0.07$ \\
\hline \multirow[t]{3}{*}{15 Jun. 2001} & $1044+719$ & 2275 & 0.36 & 0.36 & $<0.02 \pm 0.02$ \\
\hline & M $81 *$ & 188.1 & 2.16 & 0.38 & $1.15 \pm 0.20 \pm 0.06$ \\
\hline & $1053+704$ & 1354.7 & 8.28 & 0.75 & $0.61 \pm 0.06 \pm 0.07$ \\
\hline \multirow[t]{3}{*}{30 Jun. 2001} & $1044+719$ & 1669.9 & 0.43 & 0.43 & $<0.03 \pm 0.03$ \\
\hline & M $81 *$ & 257.4 & 3.14 & 0.4 & $1.22 \pm 0.16 \pm 0.07$ \\
\hline & $1053+704$ & 1051.5 & 2.61 & 0.66 & $<0.25 \pm 0.06 \pm 0.09$ \\
\hline \multirow[t]{3}{*}{ 04 Jul. 2001} & $1044+719$ & 1955.8 & 1.67 & 1.67 & $<0.09 \pm 0.09$ \\
\hline & M $81 *$ & 221.2 & 3.66 & 0.46 & $1.66 \pm 0.21 \pm 0.06$ \\
\hline & $1053+704$ & 1162.1 & 4.9 & 0.88 & $0.42 \pm 0.08 \pm 0.07$ \\
\hline \multirow[t]{3}{*}{ 19 Jul. 2001} & $1044+719$ & 1663.3 & 0.58 & 0.58 & $<0.03 \pm 0.03$ \\
\hline & M $81 *$ & 123.3 & 1.65 & 0.68 & $<1.34 \pm 0.55 \pm 0.09$ \\
\hline & $1053+704$ & 1011.9 & 1.76 & 1.05 & $<0.17 \pm 0.10 \pm 0.12$ \\
\hline \multirow[t]{3}{*}{04 Aug. 2001} & $1044+719$ & 1663 & 0.29 & 1.14 & $<0.02 \pm 0.07$ \\
\hline & M $81 *$ & 113.4 & 0.36 & 0.36 & $<0.31 \pm 0.31 \pm 0.09$ \\
\hline & $1053+704$ & 973.4 & 6.42 & 1.92 & $<0.66 \pm 0.20 \pm 0.12$ \\
\hline \multirow[t]{3}{*}{16 Aug. 2001} & $1044+719$ & 1876 & 0.51 & 0.51 & $<0.03 \pm 0.03$ \\
\hline & M $81 *$ & 107 & 0.38 & 0.42 & $<0.35 \pm 0.39 \pm 0.09$ \\
\hline & $1053+704$ & 1043.9 & 3.42 & 1.37 & $<0.33 \pm 0.13 \pm 0.12$ \\
\hline \multirow[t]{3}{*}{18 Aug. 2001} & $1044+719$ & 1496.6 & 0.46 & 0.98 & $<0.03 \pm 0.07$ \\
\hline & M $81 *$ & 79.3 & 0.2 & 0.2 & $<0.25 \pm 0.25 \pm 0.09$ \\
\hline & $1053+704$ & 830.4 & 2.44 & 0.95 & $<0.29 \pm 0.11 \pm 0.12$ \\
\hline \multirow[t]{3}{*}{25 Aug. 2001} & $1044+719$ & 1378 & 0.49 & 0.49 & $<0.04 \pm 0.04$ \\
\hline & M $81 *$ & 82.9 & 0.47 & 0.76 & $<0.56 \pm 0.56 \pm 0.09$ \\
\hline & $1053+704$ & 756.7 & 0.82 & 1.65 & $<0.11 \pm 0.22 \pm 0.12$ \\
\hline \multirow[t]{3}{*}{30 Aug. 2001} & $1044+719$ & 1846.3 & 0.76 & 0.76 & $<0.04 \pm 0.04$ \\
\hline & M $81 *$ & 104.7 & 0.54 & 0.53 & $<0.52 \pm 0.52 \pm 0.09$ \\
\hline & $1053+704$ & 988.9 & 0.41 & 0.41 & $<0.04 \pm 0.04 \pm 0.12$ \\
\hline
\end{tabular}


A. Brunthaler et al.: Radio linear and circular polarization from M 81*, Online Material p 5

Table A.4. Polarized and total flux density of M 81* at high frequencies unsing the VLA (15, 22, and $43 \mathrm{GHz})$ and BIMA (83, 86, and 230 GHz).

\begin{tabular}{lccrrrrr}
\hline \hline Date & $\begin{array}{c}\text { Frequency } \\
{[\mathrm{GHz}]}\end{array}$ & Sideband & $\begin{array}{r}I \\
{[\mathrm{mJy}]}\end{array}$ & $\begin{array}{r}Q \\
{[\mathrm{mJy}]}\end{array}$ & $\begin{array}{r}U \\
{[\mathrm{mJy}]}\end{array}$ & $\begin{array}{r}V \\
{[\mathrm{mJy}]}\end{array}$ & $\begin{array}{r}\mathrm{m}_{p} \\
{[\%]}\end{array}$ \\
\hline 09 Aug. 2003 & 15 & & $125.9 \pm 1.5$ & $<1.4$ & $<1.4$ & $<1.4$ & $<1.0$ \\
& 22 & & $118.2 \pm 1.3$ & $<1.1$ & $<1.1$ & $<1.3$ & $<1.0$ \\
& 43 & & $66.8 \pm 2.0$ & $<3.3$ & $<3.3$ & $<4.2$ & $<4.9$ \\
\hline 07 Sep. 2003 & 83 & lsb & $44.0 \pm 2.6$ & $-2.0 \pm 2.6$ & $5.6 \pm 2.6$ & $0.3 \pm 2.6$ & \\
& 86 & usb & $41.8 \pm 2.6$ & $-1.8 \pm 2.6$ & $-3.4 \pm 2.6$ & $3.2 \pm 2.6$ & \\
& & avg & $42.9 \pm 1.8$ & $-1.8 \pm 1.8$ & $1.1 \pm 1.8$ & $1.8 \pm 1.8$ & $5.1 \pm 4.2$ \\
\hline 12 Sep. 2003 & 83 & lsb & $89.4 \pm 1.8$ & $-1.3 \pm 1.8$ & $-2.1 \pm 1.8$ & $5.7 \pm 1.8$ & \\
& 86 & usb & $92.4 \pm 1.8$ & $-0.8 \pm 1.8$ & $-1.1 \pm 1.8$ & $2.5 \pm 1.8$ & \\
& & avg & $90.9 \pm 1.3$ & $-1.1 \pm 1.3$ & $-1.6 \pm 1.3$ & $4.1 \pm 1.3$ & $2.1 \pm 1.4$ \\
\hline 21 Sep. 2003 & 83 & lsb & $86.4 \pm 1.5$ & $-0.2 \pm 1.5$ & $-0.7 \pm 1.5$ & $3.8 \pm 1.5$ & \\
& 86 & usb & $86.9 \pm 1.5$ & $-1.3 \pm 1.5$ & $-0.2 \pm 1.5$ & $3.4 \pm 1.5$ & \\
& & avg & $86.7 \pm 1.1$ & $-0.2 \pm 1.1$ & $-0.5 \pm 1.1$ & $3.6 \pm 1.1$ & $0.6 \pm 1.3$ \\
\hline 06 Oct. 2003 & 83 & lsb & $70.4 \pm 2.0$ & $3.5 \pm 2.0$ & $0.7 \pm 2.0$ & $0.2 \pm 2.0$ & \\
& 86 & usb & $72.7 \pm 2.0$ & $-0.3 \pm 2.0$ & $0.3 \pm 2.0$ & $3.5 \pm 2.0$ & \\
& & avg & $71.6 \pm 1.4$ & $1.6 \pm 1.4$ & $0.5 \pm 1.4$ & $1.8 \pm 1.4$ & $2.3 \pm 2.0$ \\
\hline 09 Oct. 2003 & 83 & lsb & $46.1 \pm 1.8$ & $5.1 \pm 1.8$ & $-2.1 \pm 1.8$ & $1.0 \pm 1.8$ & \\
& 86 & usb & $45.1 \pm 1.8$ & $-3.2 \pm 1.8$ & $2.7 \pm 1.8$ & $1.5 \pm 1.8$ & \\
& & avg & $45.6 \pm 1.3$ & $1.0 \pm 1.3$ & $0.3 \pm 1.3$ & $1.2 \pm 1.3$ & $2.2 \pm 2.9$ \\
\hline 12 Oct. 2003 & 83 & lsb & $43.9 \pm 1.5$ & $-2.4 \pm 1.5$ & $-1.0 \pm 1.5$ & $3.8 \pm 1.5$ & \\
& 86 & usb & $34.5 \pm 1.5$ & $6.1 \pm 1.5$ & $4.9 \pm 1.5$ & $-1.0 \pm 1.5$ & \\
& & avg & $39.2 \pm 1.1$ & $1.9 \pm 1.1$ & $2.0 \pm 1.1$ & $1.4 \pm 1.1$ & $7.0 \pm 2.8$ \\
\hline 01 Nov. 2003 & 230 & lsb & $33.7 \pm 6$ & $-6.6 \pm 6$ & $1.2 \pm 6$ & $-4.5 \pm 6$ & \\
& & usb & $27.5 \pm 6$ & $2.7 \pm 6$ & $-19.9 \pm 6$ & $7.3 \pm 6$ & \\
& & avg & $30.6 \pm 4$ & $-2.0 \pm 4$ & $-9.4 \pm 4$ & $1.4 \pm 4$ & $31.4 \pm 13$ \\
\hline
\end{tabular}

